\title{
ESTUDO COMPARATIVO SOBRE O DESEMPENHO PERCEPTUAL E MOTOR NA IDADE ESCOLAR EM CRIANÇAS NASCIDAS PRÉ-TERMO E A TERMO
}

\author{
Lívia de Castro Magalhães , Patrícia Wendling Catarina², Vanêssa Maziero Barbosa², \\ Marisa Cota Mancinit, Maria Lúcia Paixão ${ }^{5}$
}

\begin{abstract}
RESUMO - O objetivo do estudo foi comparar o desempenho perceptual-motor na idade escolar de crianças nascidas pré-termo e a termo. Participaram do estudo 2 grupos de crianças, com idades entre 5 e 7 anos. 0 grupo I foi constituído por 35 crianças, de famílias de baixa renda, nascidas até a 34a semana de gestação e/ ou peso abaixo de $1500 \mathrm{~g}$, sem sinais de sequela neuromotora. O Grupo II foi constituído por 35 crianças nascidas a termo, com idade, sexo e nível sócio-econômico equivalentes às crianças do Grupo I. Foram aplicados os testes de Bender, acuidade motora, provas de equilíbrio e tônus postural. As crianças pré-termo obtiveram escores significativamente inferiores na maioria dos testes. Tais resultados reafirmam a importância do acompanhamento da criança pré-termo até a idade escolar e indicam a necessidade de se estimular o controle postural e a coordenação motora fina, mesmo naquelas crianças que não apresentam sequelas neurológicas evidentes.
\end{abstract}

PALAVRAS-CHAVE: prematuridade, recém nascido pré-termo, coordenação visomotora, desenvolvimento infantil, acuidade motora.

\begin{abstract}
A comparative study of the perceptual and motor performance at school age of preterm and full term children
ABSTRACT - The objective of this study was to compare the perceptualmotor performance in school age children who were born preterm and full term. Two groups of children, ages 5 to 7 , participated in this study. Group I had 35 children, from low-income families, born up to the 34 week of gestation and/or weight bellow $1500 \mathrm{~g}$. Group II had 35 full-term children, matched by age, gender and socioeconomic status to the children in Group I. Children were tested on the Bender gestalt, the motor accuracy test and on balance and postural responses measures. The preterm group obtained significantly lower scores in the majority of the tests. These besides reinforcing the importance of the follow-up of preterm children up to school age, also indicate the need to stimulate the fine motor and postural control Devment, even among preterm children who do not show evidence of neurological impairment.
\end{abstract}

KEY WORDS: prematurity, preterm infants, visuomotor coordination, child development, motor accuracy.

Embora a literatura aponte para uma maior frequência de distúrbios do desenvolvimento em crianças com história de prematuridade ${ }^{1-3}$, as controvérsias ainda são muitas e não existe um consenso quanto às características específicas dos desvios neuropsicomotores e a extensão dos déficits apresentados a longo prazo por essas crianças ${ }^{4,5}$. Um fator que contribui para as controvérsias na área é o fato de na literatura haver confusão entre baixo peso e prematuridade, sendo difícil comparar os achados dos diferentes trabalhos publicados. Apesar das controvérsias, parece evidente que quanto maior o número de fatores de risco (i.e., peso abaixo do esperado para a idade, intercorrências clínicas e longa permanência no cuidado intensivo neonatal), maiores serão as chances da criança vir a apresentar sequelas neurológicas e cognitivas, mas esta relação nem sempre é direta ${ }^{6-9}$. Sabe-se também, que na ausência de sinais severos, como paralisia cerebral e retardo mental, um número significativo de crianças com história de prematuridade vem a apresentar sinais de distúrbio de aprendizagem, dificuldades de linguagem, problemas de comportamento, déficits na coordenação motora e percepção visoespacial na idade escolar ${ }^{1-3,10,11}$.

Escola de Educação Física, Fisioterapia e Terapia Ocupacional, Universidade Federal de Minas Gerais (DTO/UFMG), Belo Horizonte MG, Brasil: ${ }^{1}$ Terapeuta Ocupacional, Doutora em Educação, Professora Adjunta do Departamento de Terapia Ocupacional DTO/UFMG; ${ }^{2}$ Terapeuta Ocupacional, Bolsista de Iniciação Científica/CNPq na época da condução do estudo; ${ }^{3}$ Terapeuta Ocupacional, Mestre em Terapia Ocupacional, Professora Assistente do DTO/UFMG; ${ }^{4}$ Terapeuta Ocupacional, Doutora em Ciências, Professora Adjunta do DTO/UFMG; ${ }^{5}$ Fisioterapeuta, Especialista no Método Neuroevolutivo, Professora Assistente do Departamento de Fisioterapia, UFMG. Estudo financiado pelo CNPq. 
Os estudos que apontam para dificuldades motoras relacionadas à prematuridade, indicam que faIhas na coordenação motora fina, observada em grupos de crianças pré-termo, podem resultar em dificuldades no manejo de objetos que exijam uma maior destreza manual, comprometendo tanto a escrita e o desempenho acadêmico, quanto a execução das atividades de vida diária ${ }^{11,12}$. Outros estudos indicam que crianças que nascem com muito baixo peso são mais propensas a déficits visuais e de linguagem, sendo que, esses problemas, associados a dificuldades percepto-motoras, podem comprometer o desempenho escolar da criança $a^{1,10,12,13}$. Em estudo mais recente, com crianças nascidas abaixo de 32 semanas, Luoma, Herrgard e Martikainen ${ }^{14}$ apontam para a grande heterogeneidade das crianças pré-termo, sendo que nesse estudo o grupo de crianças pequenas para a idade gestacional e de menor peso, foram as que receberam escores mais baixos nos testes aplicados. Estes autores não encontraram diferenças de desempenho entre crianças nascidas prétermo e a termo, nos testes de inteligência e função verbal, mas as diferenças foram significativas nas áreas de coordenação motora, controle motor fino das mãos, percepção viso-espacial e sensação tátil/ cinestésica. As autoras chamam a atenção para distúrbios sutis no planejamento motor, possivelmente relacionados à pobre precisão das percepções táteis e cinestésicas.

Além de aspectos sensório-motores, a prematuridade parece também influenciar o comportamento da criança. Segundo Marlow e colaboradores ${ }^{3}$, déficits de atenção são mais frequentes em crianças prematuras, o que pode resultar em pobre desempenho percepto-motor e dificuldades escolares. Há evidências de que, especialmente os meninos, podem apresentar um comportamento mais agressivo, hiperativo, ansioso e às vezes delinquente ${ }^{15}$. Como indicado no trabalho de Levy-Shift e colaboradores $^{16}$, com adolescentes de 13 e 14 anos com história de prematuridade, as desvantagem em testes de inteligência e coordenação visomotora, além dos sinais de hiperatividade, persistem pelo menos até a adolescência. Apesar de grande número de estudos apontar para déficits perceptuais e motores, associados à distúrbio de atenção e comportamento, na criança pré-termo, alguns autores reafirmam, principalmente com relação ao desenvolvimento cognitivo, que os fatores ambientais, em especial o baixo nível sócio-econômico, seriam decisivos, sugerindo que a medida que a criança pré-termo cresce, fatores ambientais teriam mais importância que os sinais neonatais de risco biológico ${ }^{4}$. Essa visão é compartilhada por outros autores, que sugerem um modelo interativo, em que fatores biológicos, no caso a prematuridade, e sociais se combinariam no processo de desenvolvimento da criança ${ }^{17}$.

Independente da perspectiva adotada, mais biológica ou social, são muitas as evidências de que crianças pré-termo estão sob maior risco para apresentar atraso perceptual, motor e cognitivo, associado ou não a problemas de comportamento e déficit de atenção. Como todas as áreas citadas influenciam o desempenho escolar, como medida preventiva, recomenda-se o acompanhamento do desenvolvimento dessas crianças, pelo menos, até a idade esco$\operatorname{lar}^{1,11,13,15}$. Apesar da literatura internacional ser rica em trabalhos sobre o acompanhamento e estimulação do desenvolvimento infantil, no Brasil, ainda são poucos os centros que fazem acompanhamento longitudinal sistemático de crianças pré-termo. Criado em 1988, no complexo hospitalar da Universidade Federal de Minas Gerais - UFMG, o Ambulatório da Criança de Risco (ACRIAR) conta com uma equipe interdisciplinar, composta por fisioterapeutas, fonoaudiológos, neuropediatras, pediatras e terapeutas ocupacionais, que vem se dedicando à coleta de dados longitudinais sobre o desenvolvimento e crescimento da criança pré-termo. As crianças são acompanhadas do nascimento aos 7 anos de idade, com os objetivos de: (a) detectar precocemente possíveis desvios ou atrasos no desenvolvimento, (b) orientar os pais sobre as características da criança pré-termo, (c) ensinar princípios básicos de estimulação do desenvolvimento infantil e (d) coletar dados sobre o crescimento e desenvolvimento das crianças acompanhadas.

Em estudo anterior realizado no ACRIAR ${ }^{17}$, examinamos aspectos do desenvolvimento perceptual e motor, do comportamento e do desempenho escolar em um grupo de 22 crianças pré-termo, que foram acompanhadas do nascimento até à idade préescolar. As famílias foram entrevistadas sobre as características de comportamento e desempenho escolar da criança, sendo também aplicados testes nas áreas de coordenação visomotora - testes de acuidade motora de Ayres $^{18}$ e Bender gestáltico ${ }^{19}$ - e controle postural, com provas de equilíbrio e tônus postural ${ }^{18}$. Os resultados desse estudo deram suporte a literatura na área, sendo observado que $63 \%$ das crianças da amostragem apresentavam desempenho inconsistente ou nitidamente abaixo do esperado para a idade nos testes aplicados. Apesar de relevantes, esses resultados não podem ser generalizados, devido a limitações no estudo, dentre elas, a ausência de grupo controle e as características da amostragem, na qual predominavam cri- 
anças pré-termo de famílias de baixa renda. Ou seja, além dos problemas específicos da prematuridade, essas crianças possivelmente também estavam expostas a ambientes empobrecidos e a condições, como a desnutrição e o estresse familiar, que também podem influenciar o desenvolvimento infantil ${ }^{15}$.

Este estudo tem como objetivo dar continuidade ao trabalho anterior, citado acima, procurando, através da comparação com um grupo controle, identificar possíveis diferenças no desenvolvimento entre crianças nascidas pré-termo e a termo. Foram mantidos os mesmos testes e procedimentos utilizados anteriormente, no entanto, a amostragem de recémnascidos pré-termo foi aumentada e seu desempenho comparado a um grupo controle de crianças nascidas a termo, de mesmo nível sócio-econômico. A pergunta que norteou esse estudo foi: considerado o mesmo nível sócio-econômico, existem diferenças significativas entre crianças nascidas pré-termo e a termo no desempenho em provas de equilíbrio, tônus postural e coordenação visomotora?

\section{MÉTODO}

\section{Participantes}

Participaram deste estudo 2 grupos de crianças. O Grupo I foi composto por 35 crianças nascidas até a 34 semana de gestação (21 meninos e 14 meninas), com idades entre 5 e 7 anos, nascidas no Hospital das Clínicas da UFMG e acompanhadas no ACRIAR até a idade pré-escolar. Crianças com quadros severos já diagnosticados, tais como paralisia cerebral, retardo mental, doenças genéticas e outras patologias não associadas à prematuridade, foram excluídas da amostragem. Esse grupo incluiu crianças provenientes de famílias de baixa renda (entre 1 a 3 salários mínimos), que constitui a maioria da população atendida no Ambulatório Bias Fortes/UFMG. O critério de inclusão para participação na pesquisa foi apresentar peso ao nascimento $\leq 1500 \mathrm{~g}$ e/ou idade gestacional $\leq 34$ semanas. O Grupo II foi constituído de 35 crianças nascidas a termo e sem história de intercorrências neonatais, selecionadas por características de idade e sexo semelhantes às crianças do Grupo I. As crianças do Grupo II foram recrutadas em uma Escola Municipal da periferia da Grande Belo Horizonte (Barreiro de Cima), onde estudam crianças de nível socioeconômico semelhante ao da população atendida no ACRIAR.

\section{Instrumentação}

Foram aplicados os seguintes testes:

(a) Teste gestálico de Bender ${ }^{19}$ : teste muito conhecido no Brasil e usado para avaliar a coordenação visomotora. Consiste na cópia de 9 figuras geométricas com escore de acordo com o número de erros cometidos pela criança. Quanto mais baixo o escore melhor a coordenação visomotora. O teste de Bender foi administrado e pontuado segundo as instruções de Koppitz ${ }^{19}$. (b) Teste de acuidade motora (Motor accuracy test MAC) de Ayres ${ }^{18}$ : avalia a coordenação visomotora, examinando a acuidade com que a criança traça em cima de uma linha preta, em formato de borboleta, que circunda a folha de teste. Faz-se a medida da distância que o traçado saiu fora da linha e do tempo gasto no traçado. São dados dois escores, um para acuidade (MAC-acuidade) e outro para a combinação entre o tempo gasto na prova e a distância traçada fora da linha preta (MAC-ajustado). Quanto maior o escore, melhor o desempenho da criança.

(c) Equilíbrio estático: avalia o equilíbrio em um pé só, com os olhos abertos e fechados, com um limite máximo de tempo de 15 segundos. Este teste foi aplicado segundo instruções de Ayres ${ }^{18}$.

(d) Tônus postural: foram usadas as provas de pronoextensão e supino-flexão ${ }^{18}$, que avaliam a força ou habilidade para conseguir manter padrões globais de flexão e extensão contra a gravidade. Na prova de prono-extensão a criança deita-se em prono e extende o corpo contra a gravidade elevando cabeça, peito, braços e pernas, procurando manter apenas a barriga no chão. Na prova de supinoflexão a criança deita-se em supino, fletindo todo o corpo, procurando encostar os joelhos na testa. O desempenho é avaliado quantitativamente, cronometrando o tempo que a criança mantém a posição, até um máximo de 25 segundos, e qualitativamente, registrando a qualidade da postura mantida durante a prova.

\section{Procedimentos}

As crianças do Grupo I foram avaliadas no Ambulatório Bias Fortes/UFMG, onde funciona o ACRIAR. As crianças do Grupo II foram avaliadas na própria escola, em horários estipulados pelos professores, de forma a não interferir no desempenho escolar da criança. A avaliação foi individual e durou cerca de 30 minutos. Durante a testagem buscou-se criar um ambiente descontraído e divertido para a criança. A aplicação dos testes seguiu a seguinte ordem: prono-extensão, equilíbrio em um pé só com os olhos abertos e fechados e supino-flexão. Em seguida passou-se à mesa, para administração dos testes MAC e Bender. Todas as crianças foram testadas por bolsistas de Iniciação Científica envolvidos nas atividades do ACRIAR. Os examinadores foram treinados de acordo com as instruções de cada manual de teste e antes do início da coleta examinou-se a confiabilidade entre examinadores em cerca de $10 \%$ da amostragem (4 crianças). Os resultados indicaram congruência de escores acima de $90 \%$, com índices de correlação (Pearson r) entre examinadores variando de 0,99 a 1,00. Para garantir confiabilidade, os escores dos testes de Bender e MAC foram conferidos por dois pesquisadores.

Os pais das crianças de ambos os grupos foram esclarecidos dos objetivos da pesquisa e assinaram carta de consentimento informado dando autorização para que a criança participasse do estudo. Os testes aplicados nesse estudo fazem parte da rotina de acompanhamento do desenvolvimento do ACRIAR, sendo incluídos os dados de to- 
Tabela 1. Caracterização dos participantes.

\begin{tabular}{|c|c|c|c|}
\hline \multirow[t]{2}{*}{ Participantes } & \multicolumn{2}{|c|}{ Sexo } & \multirow{2}{*}{$\begin{array}{l}\text { Idade Média } \\
\text { Meses* }\end{array}$} \\
\hline & Mas. & Fem. & \\
\hline $\begin{array}{l}\text { Pré-termo } \\
\text { (Grupo I) }\end{array}$ & 22 & 13 & $\begin{array}{c}75,09( \pm 6,83)^{\mathrm{a}} \\
(60-93)^{\mathrm{b}}\end{array}$ \\
\hline $\begin{array}{l}\text { A Termo } \\
\text { (Grupo II) }\end{array}$ & 22 & 13 & $\begin{array}{c}74,94( \pm 6,32)^{\mathrm{a}} \\
(62-92)^{\mathrm{b}}\end{array}$ \\
\hline
\end{tabular}

*Resultado de ANOVA $(F=0,008, p=0,93)$ indica que não há diferença significativa de idade entre os grupos. ${ }^{\mathrm{a}} \mathrm{O}$ valor entre parênteses é o desvio padrão. ${ }^{\mathrm{b}}$ Amplitude de idade, em meses.

das as crianças que estavam na fase final de acompanhamento no ACRIAR. As crianças do Grupo II foram recrutadas na Escola Municipal, através de carta aos pais explicando os objetivos do estudo e solicitando sua colaboração. A carta incluía um pequeno questionário sobre as condições de nascimento da criança e local para assinatura autorizando a participação da criança no estudo. Só foram avaliadas as crianças com carta de consentimento assinada pelos pais e cujas informações indicavam nascimento à termo. $\mathrm{O}$ projeto de pesquisa foi aprovado pelo Comitê de Ética em Pesquisa -COEP/UFMG.

\section{RESULTADOS}

Os dados foram analisados usando o programa estatístico SPSS, versão 10.0. Como alguns dos testes usados não foram estandardizados para a criança brasileira, nesse estudo foram analisados apenas os escores brutos obtidos em cada teste. Inicialmente foi feita uma análise descritiva, caracterizando as médias e desvio padrão para cada grupo. Em seguida foi feito o teste de homogeneidade de variância de Levene e, apesar dos resultados terem indicado va- riância homogênea em apenas 4 (idade, Bender, MAC-acuidade e equilíbrio olhos fechados direita) das 12 variáveis (incluindo idade) em estudo, optamos por dar prosseguimento à comparação dos grupos usando ANOVA, pois segundo Hinkle, Wiersma e Jurs ${ }^{20}$, quando o tamanho das amostragens é igual, como nesse estudo, o efeito da heterogeneidade de variâncias na possibilidade de erro tipo I é mínimo.

Na Tabela 1 apresentamos a caracterização da amostragem, na qual o Grupo I foi constituído por crianças pré-termo, nascidas entre 27 a 34 semanas, com idade gestacional média de 31,17 ( $\mathrm{DP}= \pm 2,32$ ) semanas e peso ao nascimento variando de 660 a 2.120g, peso médio de 1.273,53 g (DP $= \pm 333,53)$. As crianças do Grupo II tem características de sexo e idade semelhantes ao Grupo I.

Os resultados de cada grupo, com as médias (Média) de escore nos testes, o desvio padrão (DP) e resultado do teste de significância estatística (F), são apresentados na Tabela 2. Foram encontradas diferenças significativas de desempenho entre os dois grupos em todos os testes, exceto no escore do teste de acuidade motora - MAC-acuidade. Nota-se, pelas médias de escore, que as crianças nascidas à termo tiveram melhor desempenho na maioria dos testes, devendo-se observar que quanto maior o escore do teste de Bender, maior o número de erros cometidos pela criança.

\section{DISCUSSÃO}

Esse estudo, além de dar suporte às evidências de que crianças com história de prematuridade têm pior desempenho em testes perceptual-motores,

Tabela 2. Comparação dos escores entre crianças pré-termo a termo.

\begin{tabular}{|c|c|c|c|c|c|}
\hline \multirow[b]{2}{*}{ Testes } & \multicolumn{2}{|c|}{ Crianças pré-termo } & \multicolumn{2}{|c|}{ Crianças a termo } & \multirow[b]{2}{*}{$\mathrm{p}$} \\
\hline & Média & DP & Média & DP & \\
\hline BENDER & 11,57 & 4,74 & 8,90 & 4,20 & $0,018^{*}$ \\
\hline MAC - acuidade & 158,35 & 11,59 & 162,77 & 7,27 & 0,060 \\
\hline MAC - ajustado & 140,08 & 9,79 & 144,05 & 6,24 & $0,047^{*}$ \\
\hline Eq. olhos abertos direita & 12,00 & 4,77 & 14,49 & 1,27 & $0,004 * *$ \\
\hline Eq. olhos abertos esquerda & 11,66 & 4,69 & 13,97 & 2,09 & $0,008 * *$ \\
\hline Eq. olhos fechados direita & 4,37 & 3,99 & 6,90 & 4,30 & $0,012^{*}$ \\
\hline Eq. olhos fechados esquerda & 4,27 & 3,16 & 6,23 & 3,95 & $0,025^{*}$ \\
\hline Supino flexão - tempo & 16,66 & 8,45 & 23,37 & 4,17 & $0,000 * *$ \\
\hline Supino flexão - qualidade & 3,09 & 0,82 & 3,71 & 0,52 & $0,000 * *$ \\
\hline Prono extensão - tempo & 17,20 & 7,22 & 23,23 & 4,80 & $0,000 * *$ \\
\hline Prono extensão - qualidade & 3.09 & 0.66 & 3.37 & 0.65 & $0,023^{*}$ \\
\hline
\end{tabular}

${ }^{* *} p<0,001 ;{ }^{*} p<0,005 ;$ MAC, Teste de Acuidade Motora; Eq, equilíbrio 
acrescenta um dado novo, ainda pouco discutido na literatura, que é o desempenho inferior dessas crianças em provas de controle postural. Tais resultados chamam atenção para a importância do acompanhamento sistemático do desenvolvimento de recém-nascidos pré-termo até a idade escolar.

Como indicado na Tabela 1, foram encontradas diferenças significativas entre os grupos, com vantagem para as crianças a termo, em 10 dos 11 testes analisados. O teste de acuidade motora - MACacuidade - foi o único no qual não foram encontradas diferenças significativas, indicando que a habilidade de coordenação visomotora necessária para traçar a linha corretamente foi similar nos dois grupos. Observa-se, no entanto, que as crianças pré-termo gastam mais tempo para fazer a prova, pois há diferenças significativas entre os grupos quando se inclui o fator tempo (MAC-ajustado). Ou seja, para ter o mesmo nível de acuidade no traçado, as crianças pré-termo são mais lentas, o que pode ter impacto funcional, resultando em lentidão na escrita, com consequências no desempenho escolar.

Apesar das diferenças entre os grupos na acuidade motora só serem evidenciadas quando se considera o fator tempo, as crianças do grupo prétermo cometeram maior número de erros no teste de Bender, o que dá suporte à idéia de que essas crianças apresentam maior dificuldade na coordenação visomotora. Deve-se considerar que o teste de Bender avalia a coordenação visomotora de maneira um pouco diferente do MAC, pois acrescenta um elemento visoespacial, uma vez que a criança tem que copiar figuras geométricas com acuidade. O teste de Bender é bastante estudado no Brasil, sendo considerado um bom instrumento para predizer o desempenho escolar em crianças brasileiras ${ }^{21}$, o que aponta para a necessidade de se dar maior atenção à escolarização da criança pré-termo.

Os resultados inferiores nas provas de prono extensão, supino flexão e também de equilíbrio, sugerem que crianças pré-termo examinadas apresentam o tônus postural rebaixado em relação às crianças nascidas a termo. Apesar desse ser um dado novo na literatura na área, pois quase não existem estudos sobre controle postural na criança pré-termo, além do primeiro ou segundo ano de idade, ele não nos causa surpresa. A hipotonia dos extensores é, sabidamente, uma característica do recém nascido pré-termo, mas não existem trabalhos que documentam a evolução do tônus postural, em crianças prétermo, que não tem diagnóstico de paralisia cerebral, até a idade escolar. É curioso observar que sinais de baixo tônus ainda persistam aos 6 e 7 anos.
Mais estudos nessa área são necessários, pois alterações observadas na regulação de força muscular em bebês pré-termo, parecem ter impacto na função manual no primeiro ano de vida, com possíveis consequências para a coordenação motora e escrita na idade escolar ${ }^{11}$. Tônus postural pobre, associado à desvantagem no equilíbrio estático no grupo pré-termo, sugere maior dificuldade na movimentação e coordenação motora global nesse grupo de crianças.

Outro aspecto que merece consideração, é que examinando os dados apresentados na Tabela 2, observa-se que os desvios padrão do grupo pré-termo tendem a ser maiores, sugerindo que há maior variabilidade nos dados nesse grupo. Ou seja, o grupo pré-termo parece ser mais heterogêneo, um dado já comentado por Luoma, Hergard e Martkainen ${ }^{14}$ com relação à coordenação visomotora em crianças nascidas até a $32^{\mathrm{a}}$ semana de gestação. Segundo esses autores, crianças pequenas para idade gestacional e de muito baixo peso tiveram pior desempenho. Na amostragem em estudo, não foi possível isolar esses fatores, mas é importante que estudos futuros com amostragens maiores procurem contrastar sub-grupos de crianças prematuras, como por exemplo, as que nasceram com peso adequado versus as pequenas para idade gestacional.

Considerando que os dois grupos em estudo são compostos por crianças de famílias de baixa renda, entende-se que o fator sócio-econômico possivelmente não influenciou os resultados do estudo, permitindo concluir que a prematuridade tem impacto significativo no desenvolvimento perceptual-motor e postural da criança até a idade escolar. Apesar dessa ser uma conclusão pertinente e em acordo com a literatura na área, uma hipótese alternativa, que não é abordada nos trabalhos revisados, é que, em função da fragilidade inicial do recém-nascido pré-termo, os pais tenderiam à superproteção, o que poderia limitar as experiências sensório-motoras dessas crianças. Esse dado não foi examinado nesse estudo e merece consideração em trabalhos futuros. Salientamos, no entanto, que as crianças pré-termo avaliadas nesse estudo frequentam um programa de acompanhamento, no qual são dadas orientações sobre estimulação do desenvolvimento infantil. Pergunta-se então: crianças pré-termo, de famílias de baixa renda, que não frequentam programas de acompanhamento, teriam o mesmo nível de desempenho que as crianças acompanhadas? Esses mesmos resultados seriam encontrados em crianças de famílias de renda média e alta, que têm mais condições de estimular o desenvolvimento da criança? São muitas as questões que permeiam os estudos sobre 
o desenvolvimento da criança pré-termo, sendo necessários muitos outros estudos comparativos sobre os efeitos da prematuridade, incluindo crianças de diferentes níveis sociais e sub-grupos de crianças prematuras, caracterizados de acordo com as condições de nascimento. É importante também aumentar o número de variáveis em estudo, incluindo aspectos, tais como, desenvolvimento cognitivo, relacionamento mãe-filho, hábitos e interesses no brincar e desempenho escolar, dentre outros.

Um aspecto que gostaríamos de ressaltar, é que temos melhor conhecimento sobre a evolução de recém-nascidos pré-termo que apresentam sinais neurológicos evidentes e que recebem diagnóstico precoce de paralisia cerebral. Para essas crianças já existem serviços estruturados de assistência. Mas as crianças com sequelas leves, que vão se manifestar tardiamente com o fracasso escolar e distúrbios do comportamento, ainda não recebem a devida atenção, principalmente no sistema público de saúde. Dados objetivos, documentando sequelas funcionais nessas crianças, podem incentivar a criação, como em outros países, de programas preventivos de estimulação do desenvolvimento, para atender ao número crescente de crianças pré-termo que nascem nos hospitais brasileiros. Esses estudos, no entanto, só são possíveis com a coleta sistemática de dados, que precisa ser incentivada, para que venhamos a ter uma melhor compreensão das características do desenvolvimento de nossas crianças pré-termo.

\section{CONCLUSÃO}

Os resultados ressaltam a importância do acompanhamento longitudinal de crianças pré-termo, especialmente aquelas nascidas abaixo da $34^{a}$ semana de gestação e com menos de $1500 \mathrm{gr}$. As diferenças observadas entre os resultados dos testes das crianças pré-termo e a termo, indicam que o acompanhamento não deve se restringir aos primeiros dois anos de vida, pois essas crianças tendem a apresentar pior desempenho em testes percepto-motores e posturais na idade escolar. Esses problemas podem ter impacto no desempenho escolar da criança, sendo importante o trabalho preventivo, executado por equipes interdisciplinares, que incluam, além do pediatra e do neurologista infantil, a participação de terapeutas ocupacionais, fisioterapeutas e outros profissionais das áreas de saúde e educação infantil.

Um recomendação pertinente, frente aos dados apresentados nesse estudo, é que além de programas de detecção precoce de sequelas neuromotoras, crianças com história de prematuridade, especialmente as de menor peso e idade gestacional ao nascimento, mas que não apresentam quadros neurológi- cos evidentes, deveriam ser encaminhadas a programas de intervenção precoce. Tais programas deveriam dar ênfase à estimulação do desenvolvimento do controle postural, principalmente dos padrões de extensão contra a gravidade, da coordenação motora fina e percepção visual, visando a prevenção de transtornos perceptual-motores e dificuldades escolares, que são mais comuns entre essas crianças.

Agradecimentos - À equipe do ACRIAR, por seu constante suporte ao trabalho desenvolvido, e às crianças e famílias acompanhadas neste projeto sem as quais este trabalho não seria possível. Agradecemos ainda à Diretoria, professores, alunos e pais da Escola Municipal Ana Alves Teixeira que nos acolheram e colaboraram na coleta de dados do grupo controle.

\section{REFERÊNCIAS}

1. Calame A, Fawer CL, Claeys V, Arrazola L, Ducret S, Jaunin L. Neuro developmental outcome and school performance of very-low-birthweight infants at 8 years of age. Eur J Pediatr 1986;145:461-466.

2. Herrgard E, Luoma L, Tuppurainen K, Karjalainen S, Martikainen A NeuroDevmental profile at five years of children born at $<32$ weeks gestation. Dev Med Child Neurol 1993;35:1083-1096,

3. Marlow N, Roberts BL, Cooke WI. Motor skills in extremely low birthweight children at the age of 6 years. Arch Dis Child 1988;64:835-841.

4. Fawer CL, Besnier S, Forcada M, Buclin T, Calame A. Influence of perinatal, Devmental and environmental factors on cognitive abilities of preterm children without major impairments at 5 years. Early Hum Dev 1995;43:151-164.

5. Roth SC, Baudin J, Pezzani-Goldsmith M, Townsend J, Reynolds EOR, Stewart AL. Relation between neuroDevmental status of very preterm infants at one and eight years. Dev Med Child Neurol 1994;36:1049-1062.

6. Eilers BL, Desai NS, Wilson MA, Cunningham, MD. Classroom performance and social factors of children with birthweight of $1.250 \mathrm{gr}$ or less: follow-up at 5 to 8 years of age. Pediatrics 1996;77:203-208

7. Kitchen WH, Ford GW, Rickards AL, Lissenden JV, Ryan MM. Children of birth weight < 1000g: changing outcomes between ages 2 and 5 years. J Pediatr 1987;10:283-288.

8. Resnick MB, Carter RL, Ariet M, Bucciarelli, RL, Furlough RR, Evans JH, Currans JS, Ausbon WW. Effects of birth weight and sociodemographic variables on mental Devment of neonatal intensive care unit survivors. Am J Obstet Gynecol 1990;162:374-378.

9. Drillen CM, Thomson AJM, Burr K. Low-birthweightchildren at early schoolage: a longitudinal study Dev Med Child Neurol 1980;22:26-47.

10. Goyen TC, Lui K, Woods R. Visual-motor, visual-perceptual, and fine motor outcomes in very-low-birthweight children at 5 years. Dev Med Child Neurol 1998;40:76-81.

11. Platinga $Y$, Perdock J, Groot L. Hand function in low-risk preterm infants: its relation to muscle power regulation. Dev Med Child Neurol 1997;39:6-11.

12. Jongmans M, Mercuri E, Henderson S, Vries L, Sonksen P, Dubowitz L. Visual function of prematurely born children with and without perceptual-motor difficulties. Early Hum Dev 1996;45:73-82.

13. Hall A, Mckeod A, Counsell C, Thomson L, Mutch L. School attainment, cognitive ability and motor function in a total scottish very-lowbirthweight population at eight years: a controlled study. Dev Med Child Neurol 1995; 37:1037-1050.

14. Luoma L, Herrgard E, Martikainen A. Neuropsychological analysis of the visuomotor problems in children born preterm at $<32$ weeks of gestation: a 5-year prospective follow-up. Dev Med Child Neurol 1998;40:21-30.

15. Ross G, Lipper EG, Auld PA. Social competence and behavior problems in premature children at school age. Pediatrics 1991;86:391-397.

16. Levy-Shiff R, Einat G, Mogilner MB, Lerman M, Krikler R. Biological and environmental correlates of developmental outcome of prematurely born infants in early adolescents. J Pediatr Psychol 1994;19:63-78.

17. Magalhães LC, Barbosa VM, Lopes KC, Paixão ML. Estudo longitudinal do desenvolvimento de recém-nascidos pré-termo: avaliação na idade pré-escolar. Rev Bras Neurol 1999;35:87-93.

18. Ayres AJ. Southern California Sensory Integration Tests - Revised. Los Angeles: WPS, 1980.

19. Kopptiz EO. Teste gestáltico de Bender para crianças. 2.Ed. Porto Alegre: Artes Médicas, 1989.

20. Hinkle DE, Wiersma W, Jurs SG. Applied statistics for the behavioral sciences. Boston: Houghton Mifflin Co, 1988.

21. Bandeira DR, Hutz CS. A Contribuição dos testes DFH, Bender e Raven na predição do rendimento escolar na primeira série. Pisc Teoria Pesq 1994;10:59-72. 